\title{
Influence of Genetic Strain Background on the Magnitude of Behavioral Recovery Observed in Weaver Mutant Mice Following Bilateral Intrastriatal Grafting of Mesencephalic Cell Suspensions
}

\author{
L.C. Triarhou, J. Norton, B. Ghetti and J.N. Hingtgen \\ Indiana University School of Medicine, Indianapolis, IN 46202, USA
}

The weaver $(w v)$ mutation leads to loss of mesencephalic (DA) cells in the homozygous state. Behaviorally, weaver mutants display tremor, gait instability and toppling over to the sides after a few steps. Cell suspensions were prepared from the ventral mesencephalic flexure of E12 normal mouse embryos by mild enzymatic digestion (collagenase/dispase) and implanted bilaterally into the striata of weaver mutants to counteract the genetically-induced mesostriatal DA deficiency. Wild-type mice $(+/+)$, non-operated weaver mutants, and weaver mutants with bilateral grafts were tested in a battery of spontaneous behavioral tasks to determine recovery of functional responses. All the experiments were conducted in mice of the three conditions (normal, weaver, grafted weaver) maintained on two genetic strain backgrounds: a $\mathrm{C} 57 \mathrm{BL} / 6 \mathrm{~J}-A^{\mathrm{w}-\mathrm{J}} / A^{\mathrm{w}-\mathrm{J}} \times \mathrm{CBA} /$ CaGnLe- $A / A$ hybrid strain (referred to as $\left.\mathrm{B} 6 \mathrm{CBA}-A^{\mathrm{w}-\mathrm{J}} / A\right)$, and a $\mathrm{B} 6 \mathrm{CBA}-A^{\mathrm{w}-\mathrm{J}} / A \times \mathrm{BALB} /$ cJ hybrid strain (referred to as $\mathrm{BC}_{4}$ ). The following behavioral parameters were analyzed: (1) as an index of equilibrium, the time interval between placement on and falling off a balance rod suspended over the ground; (2) as an index of locomotor coordination, the number of times animals toppled over to the sides during a 5-min observation period; as indices of exploratory activity (3), the number of times animals crossed over squares in a 25 -square open-field matrix and (4) the number of times animals stood on their hind-limbs to scan the environment (rearings). Repeated-measures analyses of variance were carried out with two grouping factors, condition and strain, and one repeatedmeasures factor, trials. Appropriate transformations (in reciprocal or square-root scale) were introduced to remove existing correlations between means and standard deviations in the cells of the design, which would otherwise violate the assumptions required for validity of the $F$ tests in the analyses of variance.

In balance rod tests, since normal mice of both strains stayed on the beam for the total allotted time $(60 \mathrm{sec})$, variance was 0 , and they had to be excluded from the analysis; by comparing grafted and non-grafted weaver mutants, it was found that the grafts significantly improved performance only in the B6CBA strain (from $9.7 \mathrm{sec}$ to $25.0 \mathrm{sec}, P=0.025$ ). In the toppling data, normal mice had also to be excluded since they fell 0 times during the 5-min observation period regardless of strain; by comparing grafted and non-grafted weaver mice, there was a significant improvement in the grafted group $(P=0.0032)$, which was about the same in both strains (from 13.4 to 8.1 times in $\mathrm{BC}_{4}$, and from 16.7 to 6.0 times in $\mathrm{B} 6 \mathrm{CBA}$ ), as shown by a non-significant condition $\mathrm{x}$ strain interaction $(P=0.284)$. Concerning the data for numbers of crossings, in the $+/+$ condition the B6CBA strain was much more active than the $\mathrm{BC}_{4}$ strain (212.6 vs 38.3 times in total), but both strains increased their activity levels with successive trials. With introduction of the $w v$ gene, activity levels of both strains were drastically reduced, with $\mathrm{B} 6 \mathrm{CBA}$ mice being actually somewhat less active than $\mathrm{BC}_{4}$ mice (5.2 vs 8.4 times, respectively); neither strain showed much tendency to increase activity levels over trials. With bilateral transplants the activity levels were somewhat increased, with grafted B6CBA weaver mice being marginally more active than grafted $\mathrm{BC}_{4}$ weaver mice (32.7 and 16.3 times, respectively). In the rearing data, since there was 
absolutely no rearing observed in weaver animals of either strain and almost none in the grafted animals (only 3 grafted animals in each strain showed 1 or 2 rearings in the 3 successive observation periods), it may be that the only meaningful analysis must be limited to the normal animals.

We conclude that (i) introduction of BALB/cJ genome into B6CBA mice leads to differences in motoric activity in the wild-type; (ii) the $w v$ mutation is associated with defective motor be- haviors in both mouse strains; (iii) bilateral transplantation of mesencephalic cell suspensions to the weaver striatum leads to functional recovery of certain motor activities; (iv) the extent of graft-induced functional recovery is influenced by the mouse strain on which mutants are maintained.

Supported by USPHS R29-N329283 and PO1NS27613. 

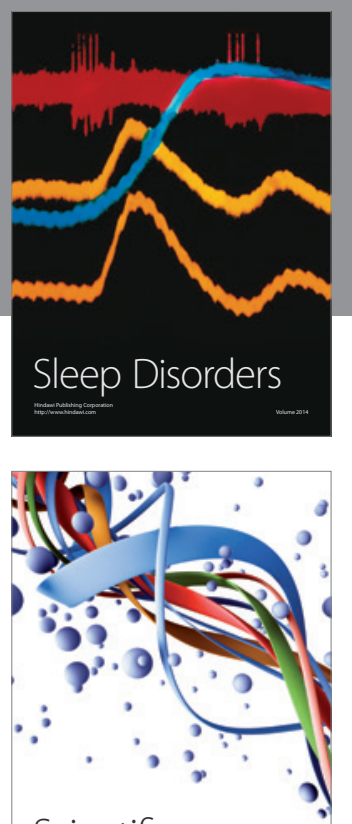

Scientifica
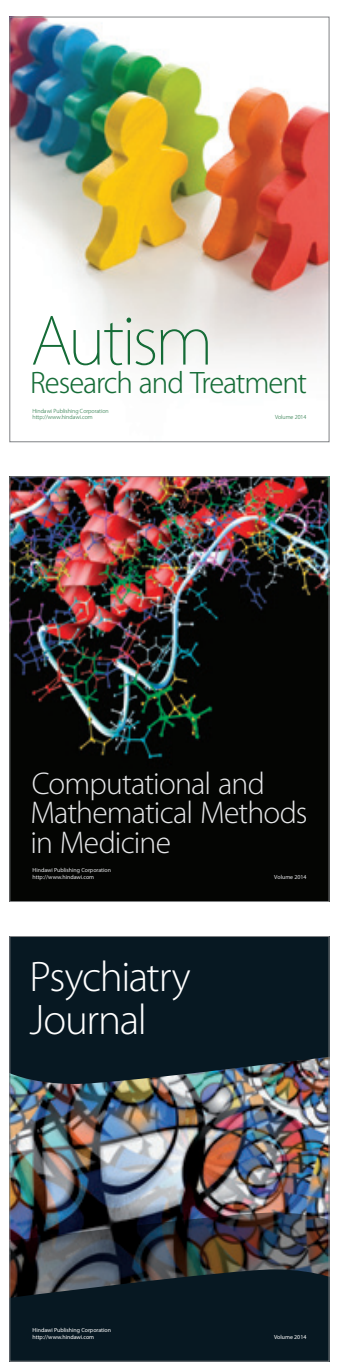
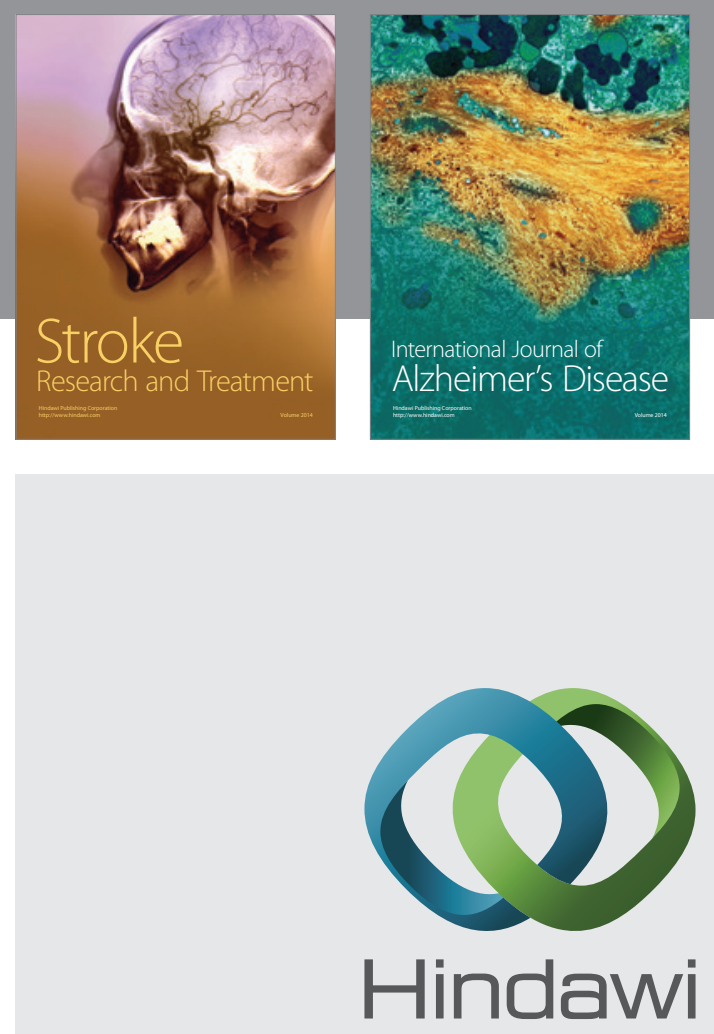

Submit your manuscripts at

http://www.hindawi.com
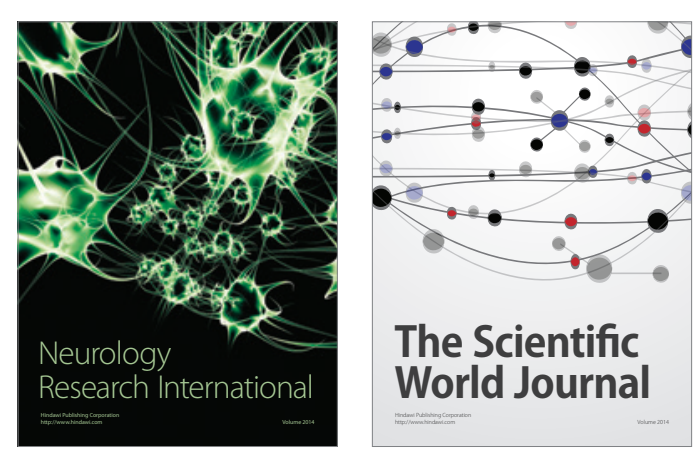

The Scientific World Journal

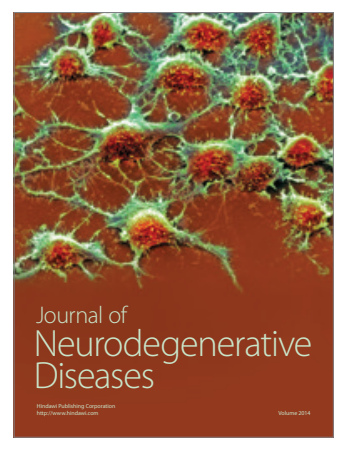

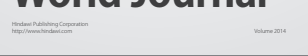

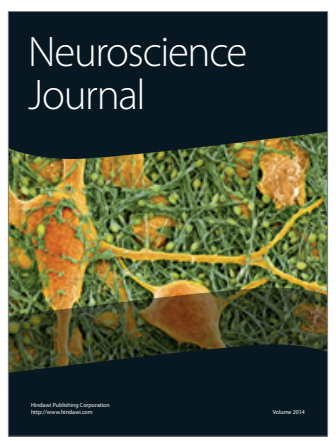

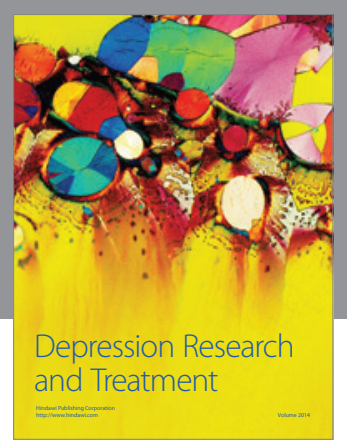
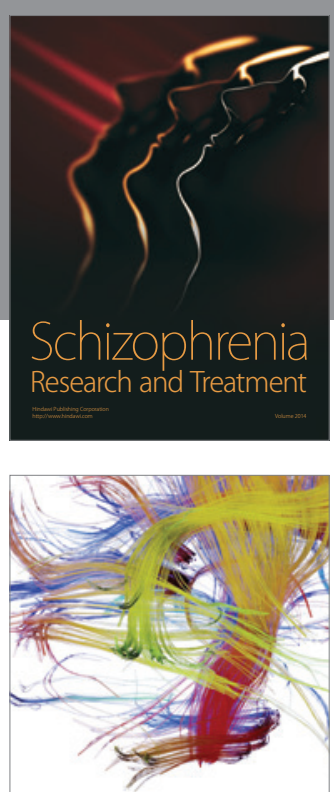

Brain Science

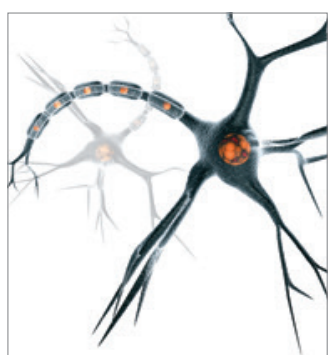

Neural Plasticity
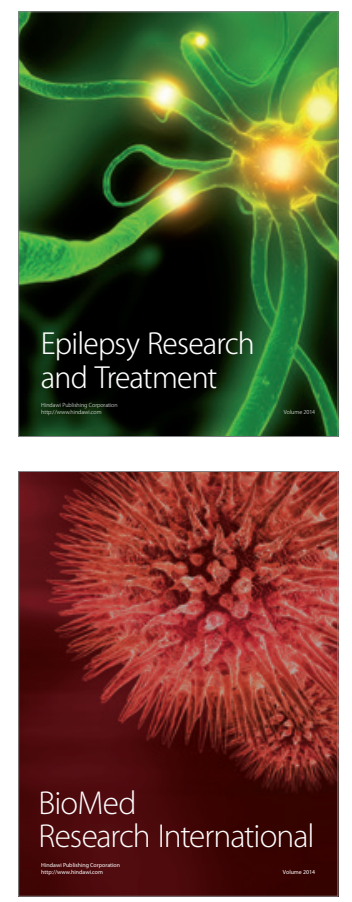

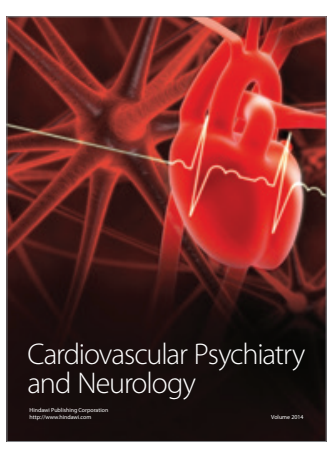

Parkinson's

Disease
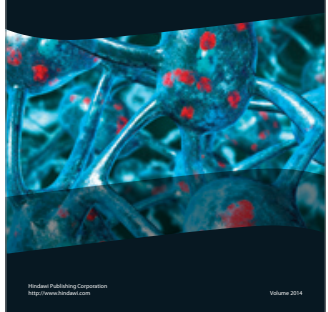